\title{
On the Hyperbolicity Constant of Line Graphs
}

\author{
Walter Carballosa José M. Rodríguez \\ Departamento de Matemáticas \\ Universidad Carlos III de Madrid \\ Av. de la Universidad 30, 28911 Leganés, Madrid, Spain \\ wcarball@math.uc3m.es, jomaro@math.uc3m.es \\ José M. Sigarreta \\ Facultad de Matemáticas \\ Universidad Autónoma de Guerrero \\ Carlos E. Adame 5, Col. La Garita, Acapulco, Guerrero, México \\ josemariasigarretaalmira@hotmail.com \\ María Villeta \\ Facultad de Matemáticas \\ Universidad Complutense de Madrid \\ Av.Puerta de Hierro s/n., 28040 Madrid, Spain \\ mvilleta@estad.ucm.es
}

Submitted: Jul 2, 2011; Accepted: Oct 14, 2011; Published: Oct 31, 2011

Mathematics Subject Classification: 05C88

\begin{abstract}
If $\mathrm{X}$ is a geodesic metric space and $x_{1}, x_{2}, x_{3} \in X$, a geodesic triangle $T=\left\{x_{1}, x_{2}, x_{3}\right\}$ is the union of the three geodesics $\left[x_{1} x_{2}\right],\left[x_{2} x_{3}\right]$ and $\left[x_{3} x_{1}\right]$ in $X$. The space $X$ is $\delta$-hyperbolic (in the Gromov sense) if any side of $T$ is contained in a $\delta$-neighborhood of the union of the two other sides, for every geodesic triangle $T$ in $X$. We denote by $\delta(X)$ the sharp hyperbolicity constant of $X$, i.e., $\delta(X):=\inf \{\delta \geq 0: X$ is $\delta$-hyperbolic $\}$. The study of hyperbolic graphs is an interesting topic since the hyperbolicity of a geodesic metric space is equivalent to the hyperbolicity of a graph related to it. The main aim of this paper is to obtain information about the hyperbolicity constant of the line graph $\mathcal{L}(G)$ in terms of parameters of the graph $G$. In particular, we prove qualitative results as the following: a graph $G$ is hyperbolic if and only if $\mathcal{L}(G)$ is hyperbolic; if $\left\{G_{n}\right\}$ is a T-decomposition of $G\left(\left\{G_{n}\right\}\right.$ are simple subgraphs of $G)$, the line graph $\mathcal{L}(G)$ is hyperbolic if and only if $\sup _{n} \delta\left(\mathcal{L}\left(G_{n}\right)\right)$ is finite. Besides, we obtain quantitative results. Two of them are quantitative versions of our qualitative results. We also prove that $g(G) / 4 \leq \delta(\mathcal{L}(G)) \leq c(G) / 4+2$,
\end{abstract}


where $g(G)$ is the girth of $G$ and $c(G)$ is its circumference. We show that $\delta(\mathcal{L}(G)) \geq \sup \{L(g): g$ is an isometric cycle in $G\} / 4$. Furthermore, we characterize the graphs $G$ with $\delta(\mathcal{L}(G))<1$.

Keywords: Infinite Graphs; Line Graphs; Connectivity; Geodesics; Hyperbolicity

\section{Introduction}

The study of mathematical properties of Gromov hyperbolic spaces and its applications is a topic of recent and increasing interest in graph theory; see, for instance $[4,5,6,7,10$, $11,14,24,25,26,27,29,31,32,35,36,39,40,41,42,44]$.

The theory of Gromov's spaces was used initially for the study of finitely generated groups (see $[16,17]$ and the references therein), where it was demonstrated to have an enormous practical importance. This theory was applied principally to the study of automatic groups (see [33]), that play an important role in sciences of the computation. Another important application of this spaces is secure transmission of information by internet (see [24, 25]). In particular, the hyperbolicity also plays an important role in the spread of viruses through the network (see [24, 25]). The hyperbolicity is also useful in the study of DNA data (see [10]).

In recent years several researchers have been interested in showing that metrics used in geometric function theory are Gromov hyperbolic. For instance, the Gehring-Osgood $j$-metric is Gromov hyperbolic; and the Vuorinen $j$-metric is not Gromov hyperbolic except in the punctured space (see [20]). The study of Gromov hyperbolicity of the quasihyperbolic and the Poincaré metrics is the subject of $[2,3,8,21,22,23,36,37,38$, 40, 41]. In particular, in [36, 40,41,42] it is proved the equivalence of the hyperbolicity of Riemann surfaces (with their Poincaré metrics) and the hyperbolicity of a simple graph; hence, it is useful to know hyperbolicity criteria for graphs.

In our study on hyperbolic graphs we use the notations of [15]. We say that $\gamma$ is a geodesic if it is an isometry, i.e., $L\left(\left.\gamma\right|_{[t, s]}\right)=d(\gamma(t), \gamma(s))=|t-s|$ for every $s, t \in[a, b]$. We say that $X$ is a geodesic metric space if for every $x, y \in X$ there exists a geodesic joining $x$ and $y$; we denote by $[x y]$ any of such geodesics (since we do not require uniqueness of geodesics, this notation is ambiguous, but it is convenient). It is clear that every geodesic metric space is path-connected. If $X$ is a graph, we use the notation $[u, v]$ for the edge of a graph joining the vertices $u$ and $v$.

In order to consider a graph $G$ as a geodesic metric space, we must identify any edge $[u, v] \in E(G)$ with the real interval $[0, l]$ (if $l:=L([u, v])$ ); hence, if we consider the edge $[u, v]$ as a graph with just one edge, then it is isometric to $[0, l]$. Therefore, any point in the interior of any edge is a point of $G$. A connected graph $G$ is naturally equipped with a distance defined on its points, induced by taking shortest paths in $G$. Then, we see $G$ as a metric graph. Along the paper we just consider simple connected graphs whose edges have length 1 and such that every vertex has finite degree; these properties guarantee that the graphs are geodesic metric spaces.

If $X$ is a geodesic metric space and $J=\left\{J_{1}, J_{2}, \ldots, J_{n}\right\}$ is a polygon, with sides 
$J_{j} \subseteq X$, we say that $J$ is $\delta$-thin if for every $x \in J_{i}$ we have that $d\left(x, \cup_{j \neq i} J_{j}\right) \leq \delta$. We denote by $\delta(J)$ the sharp thin constant of $J$, i.e., $\delta(J):=\inf \{\delta \geq 0: J$ is $\delta$-thin $\}$. If $x_{1}, x_{2}, x_{3} \in X$, a geodesic triangle $T=\left\{x_{1}, x_{2}, x_{3}\right\}$ is the union of the three geodesics $\left[x_{1} x_{2}\right],\left[x_{2} x_{3}\right]$ and $\left[x_{3} x_{1}\right]$. The space $X$ is $\delta$-hyperbolic (or satisfies the Rips condition with constant $\delta$ ) if every geodesic triangle in $X$ is $\delta$-thin. We denote by $\delta(X)$ the sharp hyperbolicity constant of $X$, i.e., $\delta(X):=\sup \{\delta(T): T$ is a geodesic triangle in $X\}$. We say that $X$ is hyperbolic if $X$ is $\delta$-hyperbolic for some $\delta \geq 0$. If $X$ is hyperbolic, then $\delta(X)=\inf \{\delta \geq 0: X$ is $\delta$-hyperbolic $\}$.

There are several definitions of Gromov hyperbolicity (see e.g. [9, 15]). These different definitions are equivalent in the sense that if $X$ is $\delta_{A}$-hyperbolic with respect to the definition $A$, then it is $\delta_{B}$-hyperbolic with respect to the definition $B$, and there exist universal constants $c_{1}, c_{2}$ such that $c_{1} \delta_{A} \leq \delta_{B} \leq c_{2} \delta_{A}$. However, for a fixed $\delta \geq 0$, the set of $\delta$-hyperbolic graphs with respect to the definition $A$, is different, in general, from the set of $\delta$-hyperbolic graphs with respect to the definition $B$. We have chosen this definition since it has a deep geometric meaning (see e.g. [15]).

The following are interesting examples of hyperbolic spaces. The real line $\mathbb{R}$ is 0 hyperbolic: in fact, any point of a geodesic triangle in the real line belongs to two sides of the triangle simultaneously, and therefore we can conclude that $\mathbb{R}$ is 0 -hyperbolic. The Euclidean plane $\mathbb{R}^{2}$ is not hyperbolic: it is clear that equilateral triangles can be drawn with arbitrarily large diameter, so that $\mathbb{R}^{2}$ with the Euclidean metric is not hyperbolic. This argument can be generalized in a similar way to higher dimensions: a normed vector space $E$ is hyperbolic if and only if $\operatorname{dim} E=1$. Every arbitrary length metric tree is 0hyperbolic: in fact, all point of a geodesic triangle in a tree belongs simultaneously to two sides of the triangle. Every bounded metric space $X$ is (diam $X$ )-hyperbolic. Every simply connected complete Riemannian manifold with sectional curvature verifying $K \leq-c^{2}$, for some positive constant $c$, is hyperbolic. We refer to $[9,15]$ for more background and further results.

We would like to point out that deciding whether or not a space is hyperbolic is usually extraordinarily difficult: Notice that, first of all, we have to consider an arbitrary geodesic triangle $T$, and calculate the minimum distance from an arbitrary point $P$ of $T$ to the union of the other two sides of the triangle to which $P$ does not belong to. And then we have to take supremum over all the possible choices for $P$ and then over all the possible choices for $T$. Without disregarding the difficulty of solving this minimax problem, notice that in general the main obstacle is that we do not know the location of geodesics in the space. Therefore, it is interesting to obtain inequalities relating the hyperbolicity constant and other parameters of graphs. Since to obtain a characterization of hyperbolic graphs is a very ambitious goal, it seems reasonable to study this problem for a particular class of graphs.

Line graphs were initially introduced in the papers [43] and [30], although the terminology of line graph was used in [19] for the first time.

The main aim of this paper is to obtain information about the hyperbolicity constant of the line graph $\mathcal{L}(G)$ in terms of parameters of the graph $G$. In particular, we prove qualitative results as the following: a graph $G$ is hyperbolic if and only if $\mathcal{L}(G)$ is hyper- 
bolic; if $\left\{G_{n}\right\}$ is a T-decomposition of $G\left(\left\{G_{n}\right\}\right.$ are simple subgraphs of $G$, see Section 3$)$, the line graph $\mathcal{L}(G)$ is hyperbolic if and only if $\sup _{n} \delta\left(\mathcal{L}\left(G_{n}\right)\right)$ is finite. Besides, we obtain quantitative results. Two of them are quantitative versions of our qualitative results.

\section{Inequalities for the hyperbolicity constant of line graphs.}

Let $\left(X, d_{X}\right)$ and $\left(Y, d_{Y}\right)$ be two metric spaces. A map $f: X \longrightarrow Y$ is said to be an $(\alpha, \beta)$-quasi-isometry, if there exist $\alpha \geq 1, \beta \geq 0$ such as for every $x, y \in X$ :

$$
\alpha^{-1} d_{X}(x, y)-\beta \leq d_{Y}(f(x), f(y)) \leq \alpha d_{X}(x, y)+\beta .
$$

Given a non-negative number $\varepsilon \geq 0$ we say that $f$ is $\varepsilon$-full if for every $y \in Y$ there exists $x \in X$ such that $d_{Y}(f(x), y) \leq \varepsilon$.

A fundamental property of hyperbolic spaces is the following (see e.g. [15, p. 88]):

Theorem 2.1 (Invariance of hyperbolicity). Let $\left(X, d_{X}\right),\left(Y, d_{Y}\right)$ be two geodesic metric spaces and $f: X \longrightarrow Y$ an $(\alpha, \beta)$-quasi-isometry.

i) If $Y$ is $\delta$-hyperbolic, then $X$ is $\delta^{\prime}$-hyperbolic, where $\delta^{\prime}$ is a constant which just depends on $\delta, \alpha$ and $\beta$.

ii) If $f$ is $\varepsilon$-full, then $X$ is hyperbolic if and only if $Y$ is hyperbolic. Furthermore, if $X$ is $\delta^{\prime}$-hyperbolic, then $Y$ is $\delta$-hyperbolic, where $\delta$ is a constant which just depends on $\delta^{\prime}, \alpha, \beta$ and $\varepsilon$.

If $G$ is a graph, we denote by $\mathcal{L}(G)$ its line graph.

Theorem 2.2. There exists a $(1 / 2)$-full $(1,1)$-quasi-isometry from $G$ on its line graph $\mathcal{L}(G)$ and, consequently, $G$ is hyperbolic if and only if $\mathcal{L}(G)$ is hyperbolic.

Furthermore, if $G$ (respectively, $\mathcal{L}(G)$ ) is $\delta$-hyperbolic, then $\mathcal{L}(G)$ (respectively, $G$ ) is $\delta^{\prime}$-hyperbolic, where $\delta^{\prime}$ is a constant which depends on $\delta$.

Proof. By Theorem 2.1, it suffices to find a $(1 / 2)$-full $(1,1)$-quasi-isometry $f: G \rightarrow \mathcal{L}(G)$. If $e \in E(G)$, we denote by $p(e)$ its corresponding vertex in $V(\mathcal{L}(G))$. We define a function $f: G \rightarrow \mathcal{L}(G)$ in the following way: if $x$ belongs to the interior of some $e \in E(G)$, then $f(x):=p(e)$; if $x \in V(G)$, let us choose some edge $e \in E(G)$ starting in $x$ and then $f(x):=p(e)$. Since $f(G)=V(\mathcal{L}(G))$, we deduce that $f$ is $(1 / 2)$-full.

Fix $x, y \in G$. If $f(x)=f(y)$, then $d_{G}(x, y) \leq 1=d_{\mathcal{L}(G)}(f(x), f(y))+1$. Let us assume that $d_{\mathcal{L}(G)}(f(x), f(y))=m$, with $m \geq 1$. Then there exist vertices $w_{0}=f(x), w_{1}, \ldots, w_{m-1}, w_{m}=f(y) \in V(\mathcal{L}(G))$ and a geodesic $\gamma:=\left[f(x), w_{1}\right] \cup$ $\left[w_{1}, w_{2}\right] \cup \cdots \cup\left[w_{m-1}, f(y)\right]$ in $\mathcal{L}(G)$ joining $f(x)$ and $f(y)$. Therefore, there exist vertices $v_{0}, v_{1}, \ldots, v_{m+1} \in V(G)$, such that $x \in\left[v_{0}, v_{1}\right], y \in\left[v_{m}, v_{m+1}\right]$ and $p\left(\left[v_{j}, v_{j+1}\right]\right)=w_{j}$ for $j=0,1, \ldots, m$. Then $d_{G}(x, y) \leq m+1=d_{\mathcal{L}(G)}(f(x), f(y))+1$. 
Let us consider now $x, y \in G \backslash V(G)$ and a geodesic $\eta:=\left[x u_{0}\right] \cup\left[u_{0}, u_{1}\right] \cup \cdots \cup$ $\left[u_{r-1}, u_{r}\right] \cup\left[u_{r} y\right]$ joining them in $G$. Then $d_{G}(x, y) \geq r$ and the path in $\mathcal{L}(G)$ given by the $r+2$ vertices $f(x), p\left(\left[u_{0}, u_{1}\right]\right), \ldots, p\left(\left[u_{r-1}, u_{r}\right]\right), f(y)$ joins $f(x)$ and $f(y)$ in $\mathcal{L}(G)$. Consequently, $d_{\mathcal{L}(G)}(f(x), f(y)) \leq r+1 \leq d_{G}(x, y)+1$. If we consider now the cases $x \in V(G)$ or $y \in V(G)$, a similar argument also gives $d_{\mathcal{L}(G)}(f(x), f(y)) \leq d_{G}(x, y)+1$.

We have obtained also a quantitative version (with explicit constants) for the hyperbolicity constants on Theorem 2.2. In order to do that, we define the Gromov product of $x, y \in G$ with base point $w \in G$ by

$$
(x, y)_{w}:=\frac{1}{2}(d(x, w)+d(y, w)-d(x, y)) .
$$

If $G$ is a Gromov hyperbolic graph, it holds

$$
(x, z)_{w} \geq \min \left\{(x, y)_{w},(y, z)_{w}\right\}-\delta
$$

for every $x, y, z, w \in G$ and some constant $\delta \geq 0$ (see e.g. [1, 15]). Let us denote by $\delta^{*}(G)$ the sharp constant for this inequality, i.e.,

$$
\delta^{*}(G):=\sup \left\{\min \left\{(x, y)_{w},(y, z)_{w}\right\}-(x, z)_{w}: x, y, z, w \in G\right\} .
$$

It is known that (2.1) is, in fact, equivalent to our definition of Gromov hyperbolicity; furthermore, we have $\delta^{*}(G) \leq 4 \delta(G)$ and $\delta(G) \leq 3 \delta^{*}(G)$ (see e.g. [1, 15]).

Theorem 2.3. For any graph $G$, we have $\delta^{*}(G)-3 \leq \delta^{*}(\mathcal{L}(G)) \leq \delta^{*}(G)+6$.

Proof. Recall that we have seen in the proof of Theorem 2.2 that there exists a (1/2)-full $(1,1)$-quasi-isometry $f: G \rightarrow \mathcal{L}(G)$.

Consequently, if $\delta^{*}(G)=\infty$, then $\delta^{*}(\mathcal{L}(G))=\infty$ and the inequalities trivially hold.

Furthermore, if $\delta^{*}(G)<\infty$, then $\delta^{*}(\mathcal{L}(G))<\infty$.

It is not difficult to check that

$$
(x, y)_{w}-\frac{3}{2} \leq(f(x), f(y))_{f(w)} \leq(x, y)_{w}+\frac{3}{2}
$$

for every $x, y, w \in G$. Then, we deduce for every $x, y, z, w \in G$,

$$
\begin{aligned}
(x, z)_{w} & \geq(f(x), f(z))_{f(w)}-\frac{3}{2} \geq \min \left\{(f(x), f(y))_{f(w)},(f(y), f(z))_{f(w)}\right\}-\delta^{*}(\mathcal{L}(G))-\frac{3}{2} \\
& \geq \min \left\{(x, y)_{w},(y, z)_{w}\right\}-\delta^{*}(\mathcal{L}(G))-3 .
\end{aligned}
$$

Then $\delta^{*}(G) \leq \delta^{*}(\mathcal{L}(G))+3$.

Furthermore, given $x^{\prime}, y^{\prime}, z^{\prime}, w^{\prime} \in \mathcal{L}(G)$ there exist $x, y, z, w \in G$ with $d_{\mathcal{L}(G)}\left(x^{\prime}, f(x)\right) \leq$ $1 / 2, d_{\mathcal{L}(G)}\left(y^{\prime}, f(y)\right) \leq 1 / 2, d_{\mathcal{L}(G)}\left(z^{\prime}, f(z)\right) \leq 1 / 2$ and $d_{\mathcal{L}(G)}\left(w^{\prime}, f(w)\right) \leq 1 / 2$. It is not difficult to check that

$$
(x, y)_{w}-3 \leq\left(x^{\prime}, y^{\prime}\right)_{w^{\prime}} \leq(x, y)_{w}+3 .
$$

Then

$$
\begin{aligned}
\left(x^{\prime}, z^{\prime}\right)_{w^{\prime}} & \geq(x, z)_{w}-3 \geq \min \left\{(x, y)_{w},(y, z)_{w}\right\}-\delta^{*}(G)-3 \\
& \geq \min \left\{\left(x^{\prime}, y^{\prime}\right)_{w^{\prime}},\left(y^{\prime}, z^{\prime}\right)_{w^{\prime}}\right\}-\delta^{*}(G)-6 .
\end{aligned}
$$

Then $\delta^{*}(\mathcal{L}(G)) \leq \delta^{*}(G)+6$. 
We deduce directly the following result.

Theorem 2.4. If $G$ is any graph, then $\frac{1}{12} \delta(G)-\frac{3}{4} \leq \delta(\mathcal{L}(G)) \leq 12 \delta(G)+18$.

Proof. Using the inequalities relating $\delta^{*}(G)$ and $\delta(G)$ and Theorem 2.3, we conclude

$$
\begin{aligned}
\delta(G) & \leq 3 \delta^{*}(G) \leq 3\left(\delta^{*}(\mathcal{L}(G))+3\right) \leq 12 \delta(\mathcal{L}(G))+9 \\
\delta(\mathcal{L}(G)) & \leq 3 \delta^{*}(\mathcal{L}(G)) \leq 3\left(\delta^{*}(G)+6\right) \leq 12 \delta(G)+18
\end{aligned}
$$

Theorem 2.5. For any graph $G$, we have $\delta(\mathcal{L}(G)) \leq \frac{1}{2} \operatorname{diam} V(G)+1$, and the inequality is sharp.

Proof. Let us consider a geodesic side $\gamma$ in any geodesic triangle $T \subset G$. Denote by $x, y$ the endpoints of $\gamma$, and by $\gamma_{1}, \gamma_{2}$ the other sides of $T$. For any $p \in \gamma$, it is clear that

$$
d\left(p, \gamma_{1} \cup \gamma_{2}\right) \leq d(p,\{x, y\}) \leq \frac{1}{2} L(\gamma) \leq \frac{1}{2} \operatorname{diam} G
$$

and consequently, $\delta(G) \leq \frac{1}{2} \operatorname{diam} G$.

Since diam $\mathcal{L}(G) \leq \operatorname{diam} V(\mathcal{L}(G))+1$ and diam $V(\mathcal{L}(G)) \leq \operatorname{diam} V(G)+1$, we conclude that $\delta(\mathcal{L}(G)) \leq \frac{1}{2} \operatorname{diam} V(G)+1$.

Note that the bound is attained when the graph is the complete graph $G=K_{6}$.

Remark 2.6. Note that, as a consequence of the proof of Theorem 2.5, we obtain that $\delta(G) \leq \frac{1}{2} \operatorname{diam} G$ for every graph $G$.

As usual, by cycle in a graph we mean a simple closed curve, i.e., a closed path with different vertices, unless the last vertex, which is equal to the first one.

A subgraph $\Gamma$ of $G$ is said isometric if $d_{\Gamma}(x, y)=d_{G}(x, y)$ for every $x, y \in \Gamma$.

The following result appears in [39, Theorem 17].

Lemma 2.7. If $G$ is any graph, then

$$
\delta(G) \geq \frac{1}{4} \sup \{L(g): g \text { is an isometric cycle in } G\} .
$$

Theorem 2.8. If $G$ is any graph, then

$$
\delta(\mathcal{L}(G)) \geq \frac{1}{4} \sup \{L(g): g \text { is an isometric cycle in } G\} .
$$

Proof. First of all, we prove that if $C$ is any isometric cycle of a graph $G$, then $p(C)$ is an isometric cycle of the line graph $\mathcal{L}(G)$.

Seeking for a contradiction, assume that $p(C)$ is not an isometric cycle of the line graph $\mathcal{L}(G)$. Then there exist two edges $e_{1}, e_{2} \in E(G)$ of $C$ such that $d_{p(C)}\left(p\left(e_{1}\right), p\left(e_{2}\right)\right)=r$ and $d_{\mathcal{L}(G)}\left(p\left(e_{1}\right), p\left(e_{2}\right)\right)=k \leq r-1$.

Since $d_{p(C)}\left(p\left(e_{1}\right), p\left(e_{2}\right)\right)=r$, we deduce that $d_{C}\left(e_{1}, e_{2}\right)=d_{G}\left(e_{1}, e_{2}\right)=r-1$. 
Since $d_{\mathcal{L}(G)}\left(p\left(e_{1}\right), p\left(e_{2}\right)\right)=k \leq r-1$, there exist edges $a_{1}, a_{2}, \ldots, a_{k-1} \in E(G)$ such that $\left[p\left(e_{1}\right), p\left(a_{1}\right)\right] \cup\left[p\left(a_{1}\right), p\left(a_{2}\right)\right] \cup \cdots \cup\left[p\left(a_{k-1}\right), p\left(e_{2}\right)\right]$ is a geodesic joining $p\left(e_{1}\right)$ and $p\left(e_{2}\right)$ in $\mathcal{L}(G)$. Therefore, $a_{1} \cup a_{2} \cup \cdots \cup a_{k-1}$ is a path joining $e_{1}$ and $e_{2}$ in $G$; this implies that $d_{G}\left(e_{1}, e_{2}\right) \leq k-1 \leq r-2<r-1=d_{G}\left(e_{1}, e_{2}\right)$, which is the contradiction we were looking for.

Now, Lemma 2.7 gives the result.

Given any graph $G$ we define, as usual, its girth $g(G)$ as the infimum of the lengths of the cycles in $G$. The following result (see [31, Theorem 17]) relates the girth of a graph and its hyperbolicity constant.

Lemma 2.9. For any graph $G$, we have $\delta(G) \geq \frac{1}{4} g(G)$, and the inequality is sharp.

Remark 2.10. One can think that the equality $\delta(G)=g(G) / 4$ holds if and only if every cycle $g$ in $G$ verifies $L(g)=g(G)$. However, this is false, as shows the following example.

Let us consider a graph $G$ obtained from a cycle graph $C_{6}$ by attaching three edges joining antipodal vertices. It is not difficult to check that $\operatorname{diam} V(G)=2, \operatorname{diam} G=2$, $\delta(G)=1, g(G)=4$, and there exists a cycle with length 6 .

Proposition 2.12 below gives a similar upper bound for $\delta(G)$. We need some preliminaries. Let us define the circumference $c(G)$ of a graph $G$ as the supremum of the lengths of its cycles.

Given any graph $G$, we denote by $\tau(G)$ the set of geodesic triangles in $G$ which are cycles and such that each one of the three vertices of the triangle is either a vertex in $V(G)$ or a midpoint of some edge in $E(G)$.

In [4] we found the following result.

Lemma 2.11. In any graph $G$, we have $\delta(G)=\sup \{\delta(T): T \in \tau(G)\}$.

Proposition 2.12. For any graph $G$, we have $\delta(G) \leq \frac{1}{4} c(G)$, and this inequality is sharp.

Proof. Let us consider any fixed geodesic triangle $T=\left\{\gamma_{1}, \gamma_{2}, \gamma_{3}\right\}$ in $G$ and $p \in T$. Without loss of generality we can assume that $p \in \gamma_{1}=[x y]$. By Lemma 2.11 we can assume that $T$ is a cycle. Since $L(T) \leq c(G)$, then $L\left(\gamma_{1}\right) \leq c(G) / 2$ and $d\left(p, \gamma_{2} \cup \gamma_{3}\right) \leq$ $d(p,\{x, y\}) \leq L\left(\gamma_{1}\right) / 2 \leq c(G) / 4$. Consequently, $\delta(G) \leq c(G) / 4$.

The bound is attained when the graph is any cycle graph with $n \geq 3$ vertices.

Proposition 2.13. For any graph $G$ which is not a tree, we have $\delta(G) \geq \frac{1}{4} g(\mathcal{L}(G))$.

Proof. Since $G$ is not a tree, we know that there is at least a cycle in $G$. By Lemma 2.9, we have $\delta(G) \geq \frac{1}{4} g(G)$. Then it suffices to note that $g(\mathcal{L}(G)) \leq g(G)$, since for every cycle in $G$ we have a cycle in $\mathcal{L}(G)$ with the same length.

The following result, which is a consequence of Theorem 2.8, is a dual version of Proposition 2.13 .

Corollary 2.14. For any graph $G$, we have $\delta(\mathcal{L}(G)) \geq \frac{1}{4} g(G)$. 
The bound in Corollary 2.14 is attained when the graph is any cycle graph with $n \geq 3$ vertices.

A matching in a finite graph $G$ is a set of edges pairwise non adjacent. An independent set in a finite graph $G$ is a set of vertices pairwise non adjacent. We denote by $M(G)$ (respectively, $I(G)$ ) the maximum of the cardinal of matching (respectively, independent) sets in $G$.

Theorem 2.15. For any finite graph $G$, we have $\delta(\mathcal{L}(G)) \leq M(G)$.

Proof. It is not difficult to check that $M(G)=I(\mathcal{L}(G))$. Let us consider a geodesic $\gamma$ in $G$ with $L(\gamma)=\operatorname{diam} V(G)$. Since $\gamma$ is a geodesic, if $v, w \in \gamma \cap V(G)$ and $d(v, w) \geq 2$, then $v w \notin E(G)$. Hence, since it is possible to choose a set of vertices $v_{1}, v_{2}, \ldots, v_{m} \in \gamma \cap V(G)$ with $d\left(v_{j}, v_{j+1}\right)=2$ and $m=\lfloor\operatorname{diam} V(G) / 2\rfloor+1$. Thus, for every graph $G$ we have $2 I(\mathcal{L}(G)) \geq \operatorname{diam} V(\mathcal{L}(G))+1 \geq \operatorname{diam} \mathcal{L}(G)$.

As a consequence of Remark 2.6 we have $\delta(\mathcal{L}(G)) \leq \frac{1}{2} \operatorname{diam} \mathcal{L}(G)$. Thus, $2 \delta(\mathcal{L}(G)) \leq$ $2 M(G)$.

Let $G$ be any graph with $\operatorname{diam} V(G) \geq 2$. We define

$$
\sigma_{2}(G):=\min \left\{\operatorname{deg}_{G}(x)+\operatorname{deg}_{G}(y): x, y \in V(G), d_{G}(x, y) \geq 2\right\} .
$$

In [34] we find the following result.

Lemma 2.16. Let $G$ be any graph with $\sigma_{2}(\mathcal{L}(G)) \geq 7$. Suppose that, for some $r \geq 3$, $\mathcal{L}(G)$ has an $r$-cycle $C$ but no $(r-1)$-cycle. Then $C$ is an isometric subgraph of $\mathcal{L}(G)$.

The following result appears in [39, Lemma 5].

Lemma 2.17. If $\Gamma$ is an isometric subgraph of $G$, then $\delta(\Gamma) \leq \delta(G)$.

Proposition 2.18. Let $G$ be any graph with $\operatorname{deg}_{G}(x)+\operatorname{deg}_{G}(y) \geq 6$ for every $[x, y] \in$ $E(G)$. Suppose that, for some $r \geq 3, \mathcal{L}(G)$ has an $r$-cycle but no $(r-1)$-cycle. Then $\delta(\mathcal{L}(G)) \geq r / 4$.

Proof. Note that if $[x, y] \in E(G)$ then $\operatorname{deg}_{\mathcal{L}(G)}(p([x, y]))=\operatorname{deg}_{G}(x)+\operatorname{deg}_{G}(y)-2 \geq 4$.

If diam $V(\mathcal{L}(G))=1$, then $\mathcal{L}(G)$ is isomorphic to a complete graph $K_{m}$ with $m \geq 5$, and $\delta(\mathcal{L}(G))=1$ (see [39, Theorem 1]); since $r=3$ is the unique value of $r$ satisfying the hypothesis, the inequality $\delta(\mathcal{L}(G)) \geq 3 / 4$ holds trivially.

If diam $V(\mathcal{L}(G)) \geq 2$, then $\sigma_{2}(\mathcal{L}(G)) \geq 8$. Therefore, if $C$ is an $r$-cycle in $\mathcal{L}(G)$, then Lemma 2.16 gives that $C$ is an isometric subgraph of $\mathcal{L}(G)$. Using that for any cycle graph $C_{n}$ with $n \geq 3$ we have $\delta\left(C_{n}\right)=L\left(C_{n}\right) / 4=n / 4$ (see [39, Theorem 1]) and Lemma 2.17 , the lower bound follows.

We deduce the following direct consequence.

Corollary 2.19. Let $G$ be any graph with $\operatorname{deg}_{G}(x) \geq 3$ for every $x \in V(G)$. Suppose that, for some $r \geq 3, \mathcal{L}(G)$ has an $r$-cycle but no $(r-1)$-cycle. Then $\delta(\mathcal{L}(G)) \geq r / 4$. 


\section{T-decompositions and consequences.}

Given a graph $G$ and a family of subgraphs $\left\{G_{n}\right\}_{n \in \Lambda}$ of $G$ verifying $\cup_{n} G_{n}=G$ and that $G_{n} \cap G_{m}$ is either a vertex or the empty set for each $n \neq m$, we define a graph $R$ as follows: for each index $n \in \Lambda$, let us consider a point $v_{n}\left(v_{n}\right.$ is an abstract point which is not contained in $G_{n}$ ) and we define the set of vertices of $R$ as $V(R)=\left\{v_{n}\right\}_{n \in \Lambda}$; two vertices of $R$ are neighbors, i.e., $\left[v_{n}, v_{m}\right] \in E(R)$ if and only if $G_{n} \cap G_{m} \neq \varnothing$. We say that the family of subgraphs $\left\{G_{n}\right\}_{n}$ of $G$ is a $T$-decomposition of $G$ if the graph $R$ is a tree.

Note that every $G_{n}$ in any T-decomposition of $G$ is an isometric subgraph of $G$.

A T-decomposition of $G$ always exists, as we will show now:

We denote by $G \backslash\{v\}$ the metric space obtained by removing the point $\{v\}$ from the metric space $G$.

We say that a vertex $v$ of a graph $G$ is a cut vertex if $G \backslash\{v\}$ is not connected. Note that any vertex with degree greater than one in a tree is a a cut vertex.

We denote by $\left\{G_{n}\right\}_{n}$ the closures in $G$ of the connected components of the set

$$
G \backslash\{v \in V(G): v \text { is a cut vertex of } G\} .
$$

It is clear that $\left\{G_{n}\right\}_{n}$ is a T-decomposition of $G$; we call it the canonical T-decomposition of $G$.

Example. Let us consider two cycle graphs $\Gamma_{1}, \Gamma_{2}$, and $x_{1} \in V\left(\Gamma_{1}\right), x_{2} \in V\left(\Gamma_{2}\right)$. Define the graph $G$ as the graph with $V(G)=V\left(\Gamma_{1}\right) \cup V\left(\Gamma_{2}\right)$ and $E(G)=E\left(\Gamma_{1}\right) \cup E\left(\Gamma_{2}\right) \cup\left[x_{1}, x_{2}\right]$. Then $\left\{\Gamma_{1}, \Gamma_{2} \cup\left[x_{1}, x_{2}\right]\right\}$ is a T-decomposition of $G$, and $\left\{\Gamma_{1}, \Gamma_{2},\left[x_{1}, x_{2}\right]\right\}$ is the canonical T-decomposition of $G$.

Consider a graph $G$ and a family of subgraphs $\left\{G_{n}\right\}_{n \in \Lambda}$ of $G$ such that $\cup_{n} G_{n}=G$ and for each $n \neq m G_{n} \cap G_{m}$ is either the empty set or an edge $e_{n m}$ such that the graph $G_{n} \cap G_{m} \backslash\left\{e_{n m}\right\}$ is not connected. We define a graph $R$ as follows: for each index $n \in \Lambda$, let us consider a point $v_{n}\left(v_{n}\right.$ is an abstract point which is not contained in $\left.G_{n}\right)$ and we define the set of vertices of $R$ as $V(R)=\left\{v_{n}\right\}_{n \in \Lambda}$; two vertices of $R$ are neighbors if and only if $G_{n} \cap G_{m} \neq \varnothing$. We say that the family of subgraphs $\left\{G_{n}\right\}_{n}$ of $G$ is a $T$-edge-decomposition of $G$ if the graph $R$ is a tree.

We will need the following result (see [6, Theorem 5]), which allows to obtain global information about the hyperbolicity of a graph from local information.

Lemma 3.1. Let $G$ be any graph and let $\left\{G_{n}\right\}_{n}$ be any T-decomposition of $G$. Then $\delta(G)=\sup _{n} \delta\left(G_{n}\right)$.

T-decompositions are a useful tool in the study of hyperbolic graphs (see e.g. [6, 31, 39]).

We have a similar result to Lemma 3.1 for $\left\{\mathcal{L}\left(G_{n}\right)\right\}_{n}$ if $\left\{G_{n}\right\}_{n}$ is a T-edgedecomposition of $G$. 
Theorem 3.2. If $\left\{G_{n}\right\}_{n}$ is any T-edge-decomposition of any graph $G$, then $\delta(\mathcal{L}(G))=$ $\sup _{n} \delta\left(\mathcal{L}\left(G_{n}\right)\right)$.

Proof. If $\left\{G_{n}\right\}_{n}$ is a T-edge-decomposition of $G$, then $\left\{\mathcal{L}\left(G_{n}\right)\right\}_{n}$ is a T-decomposition of $\mathcal{L}(G)$. Then, Lemma 3.1 gives the result.

Proposition 3.3. Let $T$ be any tree with maximum degree $\Delta$. Then

$$
\delta(\mathcal{L}(T))= \begin{cases}1, & \text { if } \Delta \geq 4, \\ 3 / 4, & \text { if } \Delta=3, \\ 0, & \text { if } \Delta \leq 2\end{cases}
$$

Proof. The canonical T-decomposition $\left\{G_{n}\right\}_{n}$ of $\mathcal{L}(T)$ has an edge for each vertex $v \in$ $V(T)$ with $\operatorname{deg}_{T}(v)=2$ and a graph isomorphic to $K_{m}$ for each vertex $v \in V(T)$ with $\operatorname{deg}_{T}(v)=m \geq 3$. Lemma 3.1 gives $\delta(\mathcal{L}(T))=\sup _{n} \delta\left(G_{n}\right)$. Besides, [39, Theorem 11] gives

$$
\delta\left(K_{m}\right)= \begin{cases}1, & \text { if } m \geq 4, \\ 3 / 4, & \text { if } m=3 .\end{cases}
$$

These facts give the result.

From [31, Proposition 5 and Theorem 7] we deduce the following results.

Lemma 3.4. If $G$ is any graph with a cycle $g$ with length $L(g) \geq 3$, then $\delta(G) \geq 3 / 4$. If there exists a cycle $g$ in $G$ with length $L(g) \geq 4$, then $\delta(G) \geq 1$.

Corollary 3.5. Let $G$ be any graph with maximum degree $\Delta$. If $\Delta \geq 3$, then $\delta(\mathcal{L}(G)) \geq$ $3 / 4$. If $\Delta \geq 4$, then $\delta(\mathcal{L}(G)) \geq 1$.

Corollary 3.6. If $G$ is any graph with a cycle $g$ with length $L(g) \geq 3$, then $\delta(\mathcal{L}(G)) \geq 3 / 4$. If there exists a cycle $g$ in $G$ with length $L(g) \geq 4$, then $\delta(\mathcal{L}(G)) \geq 1$.

In [29], the authors characterize the bridged graphs which have hyperbolicity constant 1 , for a different definition of hyperbolicity constant.

An interesting question is how to characterize the graphs $G$ with $\delta(\mathcal{L}(G))=1$, but it seems very difficult to give a description of such graphs in a simple way. However, the following theorem allows to characterize the graphs with $\delta(\mathcal{L}(G))<1$.

Theorem 3.7. If $G$ is any graph with $\delta(\mathcal{L}(G))<1$, then there are just two possibilities: $\delta(\mathcal{L}(G))=0$ or $\delta(\mathcal{L}(G))=3 / 4$. Furthermore,

- $\delta(\mathcal{L}(G))=0$ if and only if $G$ is a (finite or infinite) path graph,

- $\delta(\mathcal{L}(G))=3 / 4$ if and only if $G$ is either a tree with maximum degree $\Delta=3$ or isomorphic to $\mathrm{C}_{3}$. 
Proof. First of all, [31, Theorem 11] gives that if $\delta(\mathcal{L}(G))<1$, then we have either $\delta(\mathcal{L}(G))=0$ or $\delta(\mathcal{L}(G))=3 / 4$.

Proposition 3.3 gives that if $G$ is a path graph, then $\delta(\mathcal{L}(G))=0$.

It is well known that if $\delta(\mathcal{L}(G))=0$, then $\mathcal{L}(G)$ is a tree. Since every cycle in $G$ corresponds with a cycle in $\mathcal{L}(G)$ with the same length, $G$ is a tree. If a vertex of $G$ has degree greater or equal than 3 , then there is a cycle $g$ in $\mathcal{L}(G)$ with length $L(g) \geq 3$, and Lemma 3.4 gives that $\delta(G) \geq 3 / 4$; then the maximum degree of $G$ verifies $\Delta \leq 2$.

If $G$ is a tree with maximum degree $\Delta=3$, then Proposition 3.3 gives that $\delta(\mathcal{L}(G))=$ 3/4. If $G$ is isomorphic to $C_{3}$, then $\mathcal{L}(G)$ is also isomorphic to $C_{3}$ and $\delta\left(C_{n}\right)=L\left(C_{n}\right) / 4=$ $n / 4$ gives $\delta(\mathcal{L}(G))=3 / 4$.

If $\delta(\mathcal{L}(G))=3 / 4$, then Lemma 3.4 gives that every cycle in $\mathcal{L}(G)$ has length 3 . If a vertex of $G$ has degree greater or equal than 4 , then Corollary 3.5 gives that $\delta(\mathcal{L}(G)) \geq 1$, which is a contradiction; then the maximum degree of $G$ verifies $\Delta \leq 3$. If $G$ is a tree, then Proposition 3.3 gives $\Delta=3$ and we have the result. If $G$ has a cycle, then it has length 3 by Corollary 3.6; assume that $G$ is not isomorphic to $C_{3}$; therefore, $G$ contains a cycle isomorphic to $C_{3}$ with a vertex of degree at least 3 ; then $\mathcal{L}(G)$ contains a cycle with length at least 4 , and Lemma 3.4 gives that $\delta(\mathcal{L}(G)) \geq 1$, which is a contradiction; hence, $G$ is isomorphic to $C_{3}$.

If $\left\{G_{n}\right\}_{n}$ is a T-decomposition of $G,\left\{\mathcal{L}\left(G_{n}\right)\right\}_{n}$ is not (in general) a T-decomposition of $\mathcal{L}(G)$; however, it is possible to obtain information about $\delta(\mathcal{L}(G))$ from $\delta\left(\mathcal{L}\left(G_{n}\right)\right.$ ).

Theorem 3.8. If $\left\{G_{n}\right\}_{n}$ is any T-decomposition of any graph $G$, then

$$
\sup _{n} \delta\left(\mathcal{L}\left(G_{n}\right)\right) \leq \delta(\mathcal{L}(G)) \leq \sup _{n} \delta\left(\mathcal{L}\left(G_{n}\right)\right)+1
$$

Proof. First of all note that if a cut vertex $v$ belongs to $G_{n_{1}}, G_{n_{2}}, \ldots, G_{n_{r}}, \operatorname{deg}_{G_{n_{j}}}(v)=d_{j}$ for $j=1, \ldots, r$, and $\operatorname{deg}_{G}(v)=d=\sum_{j=1}^{r} d_{j}$, then the set of edges starting in $v$ corresponds to a subgraph $\Gamma$ of $\mathcal{L}(G)$ isomorphic to the complete graph $K_{d}$; furthermore, the subgraph $\Gamma \cap \mathcal{L}\left(G_{n_{j}}\right)$ is isomorphic to $K_{d_{j}}$ for $j=1, \ldots, r$. Hence, for each $n, \mathcal{L}\left(G_{n}\right)$ is an isometric subgraph of $\mathcal{L}(G)$; then Lemma 2.17 gives $\sup _{n} \delta\left(\mathcal{L}\left(G_{n}\right)\right) \leq \delta(\mathcal{L}(G))$.

In order to prove the upper bound of $\delta(\mathcal{L}(G))$, let us consider any geodesic triangle $T=\left\{\gamma_{1}, \gamma_{2}, \gamma_{3}\right\}$ in $\mathcal{L}(G)$ and $p \in T$. By Lemma 2.11 we can assume that $T$ is a cycle and that each one of the three vertices of the triangle $T$ (the endpoints of $\gamma_{1}, \gamma_{2}$ and $\gamma_{3}$ ) is either a vertex in $V(\mathcal{L}(G))$ or a midpoint of some edge in $E(\mathcal{L}(G))$.

Without loss of generality we can assume that $p \in \gamma_{1}$.

Case 1. Assume first that $p \in \mathcal{L}\left(G_{m}\right)$ for some fixed $m$.

Since $\mathcal{L}\left(G_{m}\right)$ is an isometric subgraph of $\mathcal{L}(G), \gamma_{j}^{m}:=\gamma_{j} \cap \mathcal{L}\left(G_{m}\right)$ is a (connected) geodesic in $\mathcal{L}\left(G_{m}\right)$ for $j=1,2,3$. We are going to construct a geodesic triangle $T_{m}$ in $\mathcal{L}\left(G_{m}\right)$ containing $\gamma_{1}^{m}, \gamma_{2}^{m}, \gamma_{3}^{m}$. Note that $p \in \gamma_{1}^{m}$. We also have that $\gamma_{2}^{m} \cup \gamma_{3}^{m} \neq \varnothing$ since $\left\{G_{n}\right\}_{n}$ is a T-decomposition of $G$. Since $T$ is a cycle, if some endpoint $x_{j}$ of $\gamma_{j}^{m}$ is not an endpoint of $\gamma_{j}$, then there exists an edge $e_{j i} \in E\left(\mathcal{L}\left(G_{m}\right)\right)$ connecting $x_{j} \in \gamma_{j}^{m}$ with some endpoint $x_{i}$ of $\gamma_{i}^{m}(i \neq j)$. 
The vertices $x_{j}, x_{i}$ of the edge $e_{j i} \in E\left(\mathcal{L}\left(G_{m}\right)\right)$ correspond to two edges $e_{1}, e_{2} \in E\left(G_{m}\right)$ starting in a cut vertex $v \in V\left(G_{m}\right)$. Therefore, $v$ belongs to $G_{m_{1}}, \ldots, G_{m_{r}}$, with $m_{1}=m$; if $\operatorname{deg}_{G}(v)=d$, then the set of edges in $G$ starting in $v$ corresponds to a subgraph $\Gamma$ of $\mathcal{L}(G)$ isomorphic to the complete graph $K_{d}$, and $e_{j i} \in E\left(\mathcal{L}\left(G_{m}\right)\right) \cap E(\Gamma)$.

Let us denote by $U$ the closure of the connected component of $T \backslash \mathcal{L}\left(G_{m}\right)$ which joins $x_{j}$ and $x_{i}$ (the endpoints of the edge $e_{j i}$ ).

Case 1.1. Assume first that $L(U) \geq 3$.

Case 1.1.1. If there is just a vertex of the geodesic triangle $T$ in $U$, then there exist vertices $y_{j}, y_{i} \in V(\Gamma)$ with $y_{j} \neq y_{i},\left[x_{j}, y_{j}\right] \subset \gamma_{j} \cap \Gamma$ and $\left[x_{i}, y_{i}\right] \subset \gamma_{i} \cap \Gamma$. Let us define $w^{\prime}$ as the midpoint of the edge $e_{j i}, g_{j}^{m}:=\gamma_{j}^{m} \cup\left[x_{j} w^{\prime}\right]$ and $g_{i}^{m}:=\gamma_{i}^{m} \cup\left[x_{i} w^{\prime}\right]$. We will show that $g_{j}^{m}$ and $g_{i}^{m}$ are geodesics in $\mathcal{L}\left(G_{m}\right)$.

In fact, we prove that if $\gamma_{j}^{m}=\left[x_{j} z_{j}\right]$, then $d_{\mathcal{L}(G)}\left(x_{j}, z_{j}\right) \leq d_{\mathcal{L}(G)}\left(x_{i}, z_{j}\right)$. Seeking for a contradiction, assume that $d_{\mathcal{L}(G)}\left(x_{j}, z_{j}\right)>d_{\mathcal{L}(G)}\left(x_{i}, z_{j}\right)$. Then

$$
d_{\mathcal{L}(G)}\left(y_{j}, z_{j}\right) \leq d_{\mathcal{L}(G)}\left(y_{j}, x_{i}\right)+d_{\mathcal{L}(G)}\left(x_{i}, z_{j}\right)<1+d_{\mathcal{L}(G)}\left(x_{j}, z_{j}\right)=L\left(\left[y_{j}, x_{j}\right]\right)+L\left(\gamma_{j}^{m}\right),
$$

and this implies that $\gamma_{j}$ is not a geodesic. This is the contradiction we were looking for. Therefore, $d_{\mathcal{L}(G)}\left(x_{j}, z_{j}\right) \leq d_{\mathcal{L}(G)}\left(x_{i}, z_{j}\right)$.

Hence, $g_{j}^{m}$ is a geodesic in $\mathcal{L}\left(G_{m}\right)$. With a similar argument we obtain that $g_{i}^{m}$ is a geodesic in $\mathcal{L}\left(G_{m}\right)$.

Case 1.1.2. If there are two vertices of the geodesic triangle $T$ in $U$, let us define $g_{j}^{m}:=\gamma_{j}^{m}$ and $g_{i}^{m}:=\gamma_{i}^{m}$; in this case we consider as third side of $T_{m}$ the edge $e_{j i}=\left[x_{i}, x_{j}\right]$.

Note that it is not possible to have the three vertices of the geodesic triangle $T$ in $U$.

Case 1.2. Assume now that $L(U)=2$. Then $U \subset \Gamma$.

Case 1.2.1. If there is just a vertex of the geodesic triangle $T$ in $U$, let us denote by $w$ this vertex of $T$.

Case 1.2.1.1. If $w \in V(\mathcal{L}(G))$, then let us define $w^{\prime}$ as the midpoint of the edge $e_{j i}$, $g_{j}^{m}:=\gamma_{j}^{m} \cup\left[x_{j} w^{\prime}\right]$ and $g_{i}^{m}:=\gamma_{i}^{m} \cup\left[x_{i} w^{\prime}\right]$; we have that $g_{j}^{m}$ and $g_{i}^{m}$ are geodesics in $\mathcal{L}\left(G_{m}\right)$.

Case 1.2.1.2. If $w$ is a midpoint of some edge in $E(\Gamma)$, without loss of generality we can assume that it is the midpoint of $\left[x_{j}, a\right]$, with $a \in V(\Gamma)$; let us define $w^{\prime}=x_{j}$, $g_{j}^{m}:=\gamma_{j}^{m}$ and $g_{i}^{m}:=\gamma_{i}^{m} \cup\left[x_{i}, x_{j}\right]$; we have that $g_{j}^{m}$ and $g_{i}^{m}$ are geodesics in $\mathcal{L}\left(G_{m}\right)$.

Case 1.2.2. If there are two vertices of the geodesic triangle $T$ in $U$, let us define $g_{j}^{m}:=\gamma_{j}^{m}$ and $g_{i}^{m}:=\gamma_{i}^{m}$; in this case we consider as third side of $T_{m}$ the edge $e_{j i}=\left[x_{i}, x_{j}\right]$.

Note that it is not possible to have $L(U)=1$, since in this case we have $U \subset \mathcal{L}\left(G_{m}\right)$, which is a contradiction.

This process allows to replace the set $U$ (a connected component of $T \backslash \mathcal{L}\left(G_{m}\right)$ ) by a set $U^{\prime} \subset \mathcal{L}\left(G_{m}\right)$ such that $\gamma_{j}^{m} \cup \gamma_{i}^{m} \cup U^{\prime}=g_{j}^{m} \cup g_{i}^{m}$ with $g_{j}^{m}$ and $g_{i}^{m}$ geodesics in $\mathcal{L}\left(G_{m}\right)$. Since there are at most three connected components of $T \backslash \mathcal{L}\left(G_{m}\right)$, iterating this process at most three times, we obtain a geodesic triangle $T_{m}$ in $\mathcal{L}\left(G_{m}\right)$ with sides $\gamma_{1}^{m *}, \gamma_{2}^{m *}, \gamma_{3}^{m *}$, containing $\gamma_{1}^{m}, \gamma_{2}^{m}, \gamma_{3}^{m}$, respectively. 
Furthermore, $d_{\mathcal{L}(G)}\left(p, \gamma_{2} \cup \gamma_{3}\right) \leq d_{\mathcal{L}\left(G_{m}\right)}\left(p, \gamma_{2}^{m *} \cup \gamma_{3}^{m *}\right)+1 \leq \delta\left(\mathcal{L}\left(G_{m}\right)\right)+1$.

Case 2. Assume now that $p \notin \cup_{n} \mathcal{L}\left(G_{n}\right)$. Then $p$ belongs to a subgraph $\Gamma$ of $\mathcal{L}(G)$ isomorphic to the complete graph $K_{d}$. Since the distance from any vertex in $K_{d}$ to any point in $K_{d}$ is less or equal than $3 / 2$, then $d_{\mathcal{L}(G)}\left(p, \gamma_{2} \cup \gamma_{3}\right) \leq 3 / 2$.

Consequently,

$$
\delta(T) \leq \max \left\{\sup _{n} \delta\left(\mathcal{L}\left(G_{n}\right)\right)+1, \frac{3}{2}\right\} .
$$

Since $T$ is arbitrary, we conclude

$$
\delta(G) \leq \max \left\{\sup _{n} \delta\left(\mathcal{L}\left(G_{n}\right)\right)+1, \frac{3}{2}\right\} .
$$

In order to finish the proof, assume first that $G$ is a tree; Proposition 3.3 gives that $\delta(\mathcal{L}(G)) \leq 1$, and then $\delta(\mathcal{L}(G)) \leq \sup _{n} \delta\left(\mathcal{L}\left(G_{n}\right)\right)+1$.

Assume now that $G$ is not a tree; then there exists a cycle $g$ in $G$ with $L_{G}(g) \geq$ 3. Note that $g$ is in $G_{n_{0}}$ for some $n_{0}$, since $\left\{G_{n}\right\}$ is a T-decomposition of $G$. The corresponding cycle $g^{\prime}$ to $g$ in $\mathcal{L}\left(G_{n_{0}}\right)$ verifies $L_{\mathcal{L}(G)}\left(g^{\prime}\right)=L_{G}(g) \geq 3$, and Lemma 3.4 gives that $\delta\left(\mathcal{L}\left(G_{n_{0}}\right)\right) \geq 3 / 4$. Consequently, $\delta\left(\mathcal{L}\left(G_{n_{0}}\right)\right)+1>3 / 2$ and $\delta(\mathcal{L}(G)) \leq \sup _{n} \delta\left(\mathcal{L}\left(G_{n}\right)\right)+$ 1 .

The lower bound in Theorem 3.8 is attained when the graph is any cycle graph $C_{n}$ with $n \geq 3$, and the upper bound is attained when the graph is any star graph $S_{n}$ with $n \geq 5$.

Theorem 3.9. If $G$ is any graph such that each graph $G_{n}$ in its canonical T-decomposition is either a cycle or an edge, then

$$
\delta(G) \leq \delta(\mathcal{L}(G)) \leq \delta(G)+1
$$

and $\delta(G)=\frac{1}{4} \sup \{L(g): g$ is a cycle in $G\}$.

Proof. If $G$ is a tree, then we just need to check that $0 \leq \delta(\mathcal{L}(G)) \leq 1$, and this is a consequence of Proposition 3.3.

Assume now that $G$ has at least a cycle.

We prove now the formula for $\delta(G)$. Lemma 3.1 and $\delta\left(C_{n}\right)=L\left(C_{n}\right) / 4=n / 4$ give

$$
\delta(G)=\sup _{n} \delta\left(G_{n}\right)=\frac{1}{4} \sup \{L(g): g \text { is a cycle in } G\} .
$$

By hypothesis each graph $G_{n}$ is either a cycle (and then $\delta\left(G_{n}\right)=\delta\left(\mathcal{L}\left(G_{n}\right)\right)=L\left(G_{n}\right) / 4$ ) or an edge (and then $\delta\left(G_{n}\right)=\delta\left(\mathcal{L}\left(G_{n}\right)\right)=0$ ). Since $G$ has at least a cycle, there exists $n$ such that $G_{n}$ (and consequently $\mathcal{L}\left(G_{n}\right)$ ) is not a tree. These facts and Theorem 3.8 give the result. 
The lower bound in Theorem 3.9 is attained when the graph is any cycle graph $C_{n}$ with $n \geq 3$, and the upper bound is attained when the graph is any star graph $S_{n}$ with $n \geq 5$.

In particular, we can bound directly the hyperbolicity constant of the line of an unicycle graph.

Corollary 3.10. If $G$ is any unicycle graph and we denote by $g$ its cycle, then $\frac{1}{4} L(g) \leq$ $\delta(\mathcal{L}(G)) \leq \frac{1}{4} L(g)+1$

We can improve the upper bound of $\delta(\mathcal{L}(G))$ in Corollary 3.10.

Theorem 3.11. If $G$ is any unicycle graph and we denote by $g$ its cycle, then $\frac{1}{4} L(g) \leq$ $\delta(\mathcal{L}(G)) \leq \frac{1}{4} L(g)+\frac{1}{2}$.

Proof. We know that the first inequality holds from Corollary 3.10.

We prove now the second inequality. The graph $G$ is the union of $g$ and the trees $T_{1}, \ldots, T_{r}$. If we denote by $G_{0}$ the subgraph of $G$ defined as $G_{0}:=\left\{x \in G: d_{G}(x, g) \leq 1\right\}$, then $\left\{G_{0}, T_{1}, \ldots, T_{r}\right\}_{n}$ is a T-edge-decomposition of $G$, and Theorem 3.2 gives $\delta(\mathcal{L}(G))=$ $\max \left\{\delta\left(\mathcal{L}\left(G_{0}\right)\right), \delta\left(\mathcal{L}\left(T_{1}\right)\right), \ldots, \delta\left(\mathcal{L}\left(T_{r}\right)\right)\right\}$. Since Proposition 3.3 gives $\delta\left(\mathcal{L}\left(T_{j}\right)\right) \leq 1$, we have $\delta(\mathcal{L}(G)) \leq \max \left\{\delta\left(\mathcal{L}\left(G_{0}\right)\right), 1\right\}$. Since $L(g) / 4+1 / 2 \geq 3 / 4+1 / 2>1$, it suffices to prove that $\delta\left(\mathcal{L}\left(G_{0}\right)\right) \leq L(g) / 4+1 / 2$.

In order to do that, we just need to construct the geodesic triangle $T_{0}$ in $\mathcal{L}(g)$ with sides $\gamma_{1}^{0 *}, \gamma_{2}^{0 *}, \gamma_{3}^{0 *}$, following the proof of Theorem 3.8 (replacing $\mathcal{L}\left(G_{m}\right)$ by $\mathcal{L}(g)$ ). In this case, if $p \in \mathcal{L}(g)$, then $d_{\mathcal{L}\left(G_{0}\right)}\left(p, \gamma_{2} \cup \gamma_{3}\right) \leq \delta(\mathcal{L}(g))+1 / 2$. Furthermore, if $p \notin \mathcal{L}(g)$, then $d_{\mathcal{L}\left(G_{0}\right)}\left(p, \gamma_{2} \cup \gamma_{3}\right) \leq 5 / 4$. Hence, we conclude

$$
\delta\left(\mathcal{L}\left(G_{0}\right)\right) \leq \max \left\{\delta(\mathcal{L}(g))+\frac{1}{2}, \frac{5}{4}\right\}
$$

and, since $\delta(\mathcal{L}(g))=\delta(g)=L(g) / 4$, we deduce $\delta(\mathcal{L}(g))+1 / 2 \geq 3 / 4+1 / 2=5 / 4$ and

$$
\delta\left(\mathcal{L}\left(G_{0}\right)\right) \leq \delta(\mathcal{L}(g))+\frac{1}{2}=\frac{1}{4} L(g)+\frac{1}{2} .
$$

Both inequalities in Theorem 3.11 are sharp: the first one is attained when the graph is any cycle graph $C_{n}$ with $n$ vertices; the second one is attained when the graph is any cycle graph $C_{2 n}$ with two edges attached in antipodal vertices.

We also have the following result.

Theorem 3.12. If $G$ is any graph with $\delta(G)<1$, then $\delta(\mathcal{L}(G)) \leq 7 / 4$.

Proof. If $G$ is a tree, then Proposition 3.3 gives that $\delta(\mathcal{L}(G)) \leq 1<7 / 4$. Assume now that $G$ has a cycle. Since $\delta(G)<1$, Lemma 3.4 gives that every cycle $g$ in $G$ has length $L(g)=3$. Then each graph $G_{n}$ in the canonical T-decomposition of $G$ is either a cycle with length 3 or an edge, and Theorem 3.9 gives $\delta(G)=3 / 4$ and $\mathcal{L}(G) \leq 7 / 4$. 
The following theorem is a similar result to Proposition 2.12 for line graphs.

Theorem 3.13. For any graph $G$, we have $\frac{1}{4} g(G) \leq \delta(\mathcal{L}(G)) \leq \frac{1}{4} c(G)+2$.

Proof. The first inequality is just Corollary 2.14 .

In order to prove the second inequality, let us consider the canonical T-decomposition $\left\{G_{n}\right\}_{n}$ of $G$. Fix any geodesic triangle $T=\left\{\gamma_{1}, \gamma_{2}, \gamma_{3}\right\}$ in $\mathcal{L}(G)$ and $p \in T$. By Lemma 2.11 we can assume that $T$ is a cycle. Without loss of generality we can assume that $p \in \gamma_{1}$.

If $p \notin \cup_{n} \mathcal{L}\left(G_{n}\right)$, we have seen in the proof of Theorem 3.8 that $d_{\mathcal{L}(G)}\left(p, \gamma_{2} \cup \gamma_{3}\right) \leq 3 / 2$.

Case 1. Assume now that $p \in \mathcal{L}\left(G_{m}\right)$ for some fixed $m$ and $G_{m}$ contains cycles. Since $\mathcal{L}\left(G_{m}\right)$ is an isometric subgraph of $\mathcal{L}(G), \gamma_{j}^{m}:=\gamma_{j} \cap \mathcal{L}\left(G_{m}\right)$ is a (connected) geodesic in $\mathcal{L}\left(G_{m}\right)$ for $j=1,2,3$. Then $p \in \gamma_{1}^{m}=[x y]$. Note that, since $\left\{G_{n}\right\}_{n}$ is a T-decomposition in $G, 2 \operatorname{diam} G_{m} \leq c\left(G_{m}\right)$. Furthermore, $\operatorname{diam} V\left(\mathcal{L}\left(G_{m}\right)\right) \leq \operatorname{diam} V\left(G_{m}\right)+1$ and thus $\operatorname{diam} \mathcal{L}\left(G_{m}\right) \leq \operatorname{diam} G_{m}+2$. Since $d_{\mathcal{L}(G)}\left(x, \gamma_{2}^{m} \cup \gamma_{3}^{m}\right), d_{\mathcal{L}(G)}\left(y, \gamma_{2}^{m} \cup \gamma_{3}^{m}\right) \leq 1$, we deduce

$$
\begin{aligned}
d_{\mathcal{L}(G)}\left(p, \gamma_{2} \cup \gamma_{3}\right) & \leq d_{\mathcal{L}(G)}\left(p, \gamma_{2}^{m} \cup \gamma_{3}^{m}\right) \leq d_{\mathcal{L}(G)}(p,\{x, y\})+1 \\
& \leq \frac{1}{2} L\left(\gamma_{1}^{m}\right)+1 \leq \frac{1}{2} \operatorname{diam} \mathcal{L}\left(G_{m}\right)+1 \leq \frac{1}{2} \operatorname{diam} G_{m}+2 \\
& \leq \frac{1}{4} c\left(G_{m}\right)+2 \leq \frac{1}{4} c(G)+2 .
\end{aligned}
$$

Case 2. Assume now that $G_{m}$ is a tree. As a consequence of Proposition 3.3, we obtain that if $T$ is any tree, then $\delta(\mathcal{L}(T)) \leq 1$.

Therefore, in any case we have

$$
\delta(\mathcal{L}(G)) \leq \frac{1}{4} c(G)+2
$$

The lower bound in Theorem 3.13 is attained when the graph is any cycle graph with $n \geq 3$ vertices.

Next, two examples that show how accurate the upper bound in Theorem 3.13 is.

If $G$ is the star graph $G=K_{1,4}$, then $\mathcal{L}(G)=K_{4}, \delta(G)=0$ and $\delta(\mathcal{L}(G))=1=$ $c(G) / 4+1$.

If $G$ is the cycle $C_{2 n}$ with two edges attached in antipodal vertices, then $\mathcal{L}(G)$ is the cycle $C_{2 n}$ with two graphs isomorphic to $C_{3}$ attached in antipodal edges. It is not difficult to check that $\delta(G)=n / 2$ and $\delta(\mathcal{L}(G))=n / 2+1 / 2=c(G) / 4+1 / 2$.

\section{Acknowledgements}

We would like to thank the referee for a careful reading of the manuscript and for some helpful suggestions.

This work was partly supported by the Spanish Ministry of Science and Innovation through projects MTM2009-07800, MTM2008-02829-E and a grant from CONACYT (CONACYT-UAG I0110/62/10), México. 


\section{References}

[1] Alonso, J., Brady, T., Cooper, D., Delzant, T., Ferlini, V., Lustig, M., Mihalik, M., Shapiro, M., Short, H., Notes on word hyperbolic groups, in: E. Ghys, A. Haefliger, A. Verjovsky (Eds.), Group Theory from a Geometrical Viewpoint, World Scientific, Singapore, 1992.

[2] Alvarez, V., Portilla, A., Rodríguez, J. M. and Tourís, E., Gromov hyperbolicity of Denjoy domains, Geom. Dedicata 121 (2006), 221-245.

[3] Balogh, Z. M. and Buckley, S. M., Geometric characterizations of Gromov hyperbolicity, Invent. Math. 153 (2003), 261-301.

[4] Bermudo, S., Rodríguez, J. M. and Sigarreta, J. M., Computing the hyperbolicity constant. Submitted.

[5] Bermudo, S., Rodríguez, J. M., Sigarreta, J. M. and Vilaire, J.-M., Mathematical properties of Gromov hyperbolic graphs, AIP Conference Proceedings, Vol. 1281 (2010), 575-578.

[6] Bermudo, S., Rodríguez, J. M., Sigarreta, J. M. and Vilaire, J.-M., Gromov hyperbolic graphs. Submitted.

[7] Bermudo, S., Rodríguez, J. M., Sigarreta, J. M. and Tourís, E., Hyperbolicity and complement of graphs, Appl. Math. Letters 24 (2011), 1882-1887.

[8] Bonk, M., Heinonen, J. and Koskela, P., Uniformizing Gromov hyperbolic spaces. Astérisque 270 (2001).

[9] Bowditch, B. H., Notes on Gromov's hyperobolicity criterion for path-metric spaces. Group theory from a geometrical viewpoint, Trieste, 1990 (ed. E. Ghys, A. Haefliger and A. Verjovsky; World Scientific, River Edge, NJ, 1991) 64-167.

[10] Brinkmann, G., Koolen J. and Moulton, V., On the hyperbolicity of chordal graphs, Ann. Comb. 5 (2001), 61-69.

[11] Carballosa, W., Pestana, D., Rodríguez, J. M. and Sigarreta, J. M., Gromov hyperbolicity of supergraphs. Submitted.

[12] Chepoi, V., Dragan, F. F., Estellon, B., Habib, M. and Vaxes Y., Notes on diameters, centers, and approximating trees of $\delta$-hyperbolic geodesic spaces and graphs, Electr. Notes Discrete Math. 31 (2008), 231-234.

[13] Dirac, G.A., Généralisation du théorème de Menger, C .R. Acad. Sci. Paris 250 (26) (1960), 4252-4253.

[14] Frigerio, R. and Sisto, A., Characterizing hyperbolic spaces and real trees, Geom. Dedicata 142 (2009), 139-149.

[15] Ghys, E. and de la Harpe, P., Sur les Groupes Hyperboliques d'après Mikhael Gromov. Progress in Mathematics 83, Birkhäuser Boston Inc., Boston, MA, 1990.

[16] Gromov, M., Hyperbolic groups, in "Essays in group theory". Edited by S. M. Gersten, M. S. R. I. Publ. 8. Springer, 1987, 75-263. 
[17] Gromov, M. (with appendices by M. Katz, P. Pansu and S. Semmes), Metric Structures for Riemannian and Non-Riemannnian Spaces. Progress in Mathematics, vol. 152. Birkhäuser, 1999.

[18] Guold, R., Pfender, F., Pancyclicity in Claw-free Graphs, Discrete Math. 256 (2002), 151-160.

[19] Harary, F., Norman, R. Z., Some properties of line digraphs, Rend. Circ. Math. Palermo 9 (1960), 161-169.

[20] Hästö, P. A., Gromov hyperbolicity of the $j_{G}$ and $\tilde{\jmath}_{G}$ metrics, Proc. Amer. Math. Soc. 134 (2006), 1137-1142.

[21] Hästö, P. A., Lindén, H., Portilla, A., Rodríguez, J. M. and Tourís, E., Gromov hyperbolicity of Denjoy domains with hyperbolic and quasihyperbolic metrics. To appear in J. Math. Soc. Japan.

[22] Hästö, P. A., Portilla, A., Rodríguez, J. M. and Tourís, E., Gromov hyperbolic equivalence of the hyperbolic and quasihyperbolic metrics in Denjoy domains, Bull. London Math. Soc. 42 (2010), 282-294.

[23] Hästö, P. A., Portilla, A., Rodríguez, J. M. and Tourís, E., Uniformly separated sets and Gromov hyperbolicity of domains with the quasihyperbolic metric, Medit. J. Math. 8 (2011), 47-65.

[24] Jonckheere, E. A., Controle du trafic sur les reseaux a geometrie hyperbolique-Une approche mathematique a la securite de l'acheminement de l'information, J. Europ. Syst. Autom. 37(2) (2003), 145-159.

[25] Jonckheere, E. A. and Lohsoonthorn, P., Geometry of network security, American Control Conference ACC (2004), 111-151.

[26] Jonckheere, E. A., Lohsoonthorn, P. and Ariaesi, F, Upper bound on scaled Gromovhyperbolic delta, Appl. Math. Comput. 192 (2007), 191-204.

[27] Jonckheere, E. A., Lohsoonthorn, P. and Bonahon, F., Scaled Gromov hyperbolic graphs, J. Graph Theory 2 (2007), 157-180.

[28] Kanai, M., Rough isometries and combinatorial approximations of geometries of noncompact Riemannian manifolds, J. Math. Soc. Japan 37 (1985), 391-413.

[29] Koolen, J. H. and Moulton, V., Hyperbolic Bridged Graphs, Europ. J. Comb. 23 (2002), 683-699.

[30] Krausz, J., Démonstration nouvelle d'un théorème de Whitney sur les réseaux, Mat. Fiz. Lapok 50 (1943), 75-85.

[31] Michel, J., Rodríguez, J. M., Sigarreta, J. M. and Villeta, M., Hyperbolicity and parameters of graphs, Ars Comb. Volume C (2011), 43-63.

[32] Michel, J., Rodríguez, J. M., Sigarreta, J. M. and Villeta, M., Gromov hyperbolicity in cartesian product graphs, Proc. Indian Acad. Sci. Math. Sci. 120 (2010), 1-17.

[33] Oshika, K., Discrete groups, AMS Bookstore, 2002. 
[34] Pfender, F., A note on cycle spectra on line graphs, Discrete Math. 309 (2009), 2922-2924.

[35] Portilla, A., Rodríguez, J. M., Sigarreta, J. M. and Vilaire, J.-M., Gromov hyperbolic tessellation graphs. To appear in Utilitas Math.

[36] Portilla, A., Rodríguez, J. M. and Tourís, E., Gromov hyperbolicity through decomposition of metric spaces II, J. Geom. Anal. 14 (2004), 123-149.

[37] Portilla, A., Rodríguez, J. M. and Tourís, E., Stability of Gromov hyperbolicity, J. Advan. Math. Studies 2 (2009), 77-96.

[38] Portilla, A. and Tourís, E., A characterization of Gromov hyperbolicity of surfaces with variable negative curvature, Publ. Mat. 53 (2009), 83-110.

[39] Rodríguez, J. M., Sigarreta, J. M., Vilaire, J.-M. and Villeta, M., On the hyperbolicity constant in graphs, Discrete Math. 311 (2011), 211-219.

[40] Rodríguez, J. M. and Tourís, E., Gromov hyperbolicity through decomposition of metric spaces, Acta Math. Hung. 103 (2004), 53-84.

[41] Rodríguez, J. M. and Tourís, E., Gromov hyperbolicity of Riemann surfaces, Acta Math. Sinica 23 (2007), 209-228.

[42] Tourís, E., Graphs and Gromov hyperbolicity of non-constant negatively curved surfaces, J. Math. Anal. Appl. 380 (2011), 865-881.

[43] Whitney, H., Congruent graphs and the connectivity of graphs, Amer. J. Math. 54 (1932), 150-168.

[44] Wu, Y., Zhang, C., Hyperbolicity and chordality of a graph, The Electronic Journal of Combinatorics 18 (2011), paper 43, 22 pp. 